\title{
Agenesia de la arteria carótida interna
}

\section{Internal carotid artery agenesis}

Alejandro Pineda-Urias' ${ }^{*}$, Grecia Chávez-Chavira ${ }^{1}$, José R. Anglés-Rascón ${ }^{1}$,

Fernando A. Morales-Aguilar ${ }^{1}$, Leopoldo Rivera-Castaño ${ }^{2}$ y César Cardona-Martínez ${ }^{1}$

${ }^{1}$ Departamento de Radiología; ${ }^{2}$ Departamento de Neurología. Hospital Ángeles Chihuahua, Chihuahua, México

\section{RESUMEN}

La primera descripción de un caso de agenesia de la arteria carótida interna se atribuye a Tode, en 1787, durante una autopsia. En 1954, Verbiest, mediante angiografía cerebral, reporta el primer caso de esta angiopatía. Lie y Hage, en 1968, clasifican y otorgan las definiciones de agenesia, aplasia e hipoplasia. No existe una etiología establecida, por lo que se cree que hay factores durante la embriogénesis que pueden causarla. No obstante, suele ser un hallazgo incidental durante estudios de imagenología en pacientes con historia clínica de síntomas inespecíficos.

Palabras clave: Agenesia. Carótida. Angiografía. Aplasia. Hipoplasia. Embriogénesis.

\section{ABSTRACT}

The first case of internal carotid artery agenesis is attributed to Tode, in 1787, during an autopsy. In 1954, Verbiest, through a cerebral angiography, reports the first case of this angiopathy. Lie and Hage, in 1968, classifies and grants the definitions of agenesis, aplasia and hypoplasia. There is no established etiology, so it is believed that there are factors during embryogenesis that can develop it. However, it is usually an incidental finding during imaging studies in patients who have a medical history with nonspecific symptoms.

Keywords: Agenesis. Carotid. Angiography. Aplasia. Hypoplasia. Embryogenesis. 


\section{CASO CLÍNICO}

Mujer en la segunda década de la vida con antecedentes de síncope a los 12 años de edad, sin manejo ni revisión médica. Presenta de manera esporádica somnolencia e incapacidad de control de los esfínteres durante un cuadro epiléptico. Vuelve a presentar otro 1 año después, por lo que acude a consulta y se le realiza un electroencefalograma que reporta brotes de ondas teta bifrontoparietales de predominio izquierdo, y se concluye el diagnóstico de epilepsia con crisis de inicio focal motora tipo atónica de etiología desconocida. Se maneja con levetiracetam, logrando un control parcial, ya que se repiten las crisis epilépticas durante los siguientes 2 años por mala aplicación del tratamiento. La paciente acude por cuenta propia al servicio de radiología 1 año después para realizarse una resonancia magnética (RM) de encéfalo, ya que fue uno de los estudios sugeridos y no se realizó por falta de recursos económicos. En la RM se practica al mismo tiempo una angiorresonancia, que reporta una agenesia de arteria carótida interna (ACI) izquierda (Figs. 1 and 2). La paciente fue dada de alta con aumento de la dosis de levetiracetam, sin haber vuelto a presentar cuadros epilépticos hasta la actualidad.

\section{DISCUSIÓN}

La agenesia, hablando de la ACI, es la ausencia completa de la estructura vascular; la aplasia es la ausencia vascular con evidencia de un precursor embrionario; y la hipoplasia es la falta de desarrollo, por lo que puede existir o no un remanente embrionario funcional o no funcional. Otros autores concluyen estos términos como una misma alteración congénita ${ }^{1,2}$.

A raíz del descubrimiento del polígono de Willis en 1664, se sabe que existe menos de un $40 \%$ de esta parte anatómica de manera completa, como se muestra en los libros de texto, por lo que el $60 \%$ restante involucra variantes, tales como fenestraciones, duplicaciones, trifurcaciones, vasos accesorios, orígenes infundibulares y fetales, agenesias, aplasias e hipoplasia. En relación con los tres últimos, suelen compensarse con nuevas anastomosis homolaterales, contralaterales o ambas $^{2}$.

La agenesia de la ACI se presenta en menos del $0.01 \%$ de la población mundial, frecuentemente con ausencia unilateral del lado izquierdo, con una proporción 3:1 respecto al derecho.

No existe una etiología específica, aunque existe la teoría de que el estrés hemodinámico y metabólico durante la embriogénesis es producido por un plegamiento exagerado hacia algún lado del embrión, constriñendo así las bandas amnióticas ${ }^{3}$. En general ocurre entre la tercera y la quinta semanas de gestación, durante el desarrollo del tercer arco aórtico y la aorta dorsal ${ }^{4,5}$.

Clínicamente esta malformación es asintomática en las primeras dos décadas de vida, debido a la colateralidad compensatoria. En pacientes jóvenes es común la aparición de síntomas como retraso psicomotor, dislalia y somnolencia. Aunque es un hallazgo incidental, a partir de los 35-40 años de edad, sumándose a ello factores patológicos y no patológicos, como obesidad, tabaquismo, hipertensión o alguna angiopatía, 

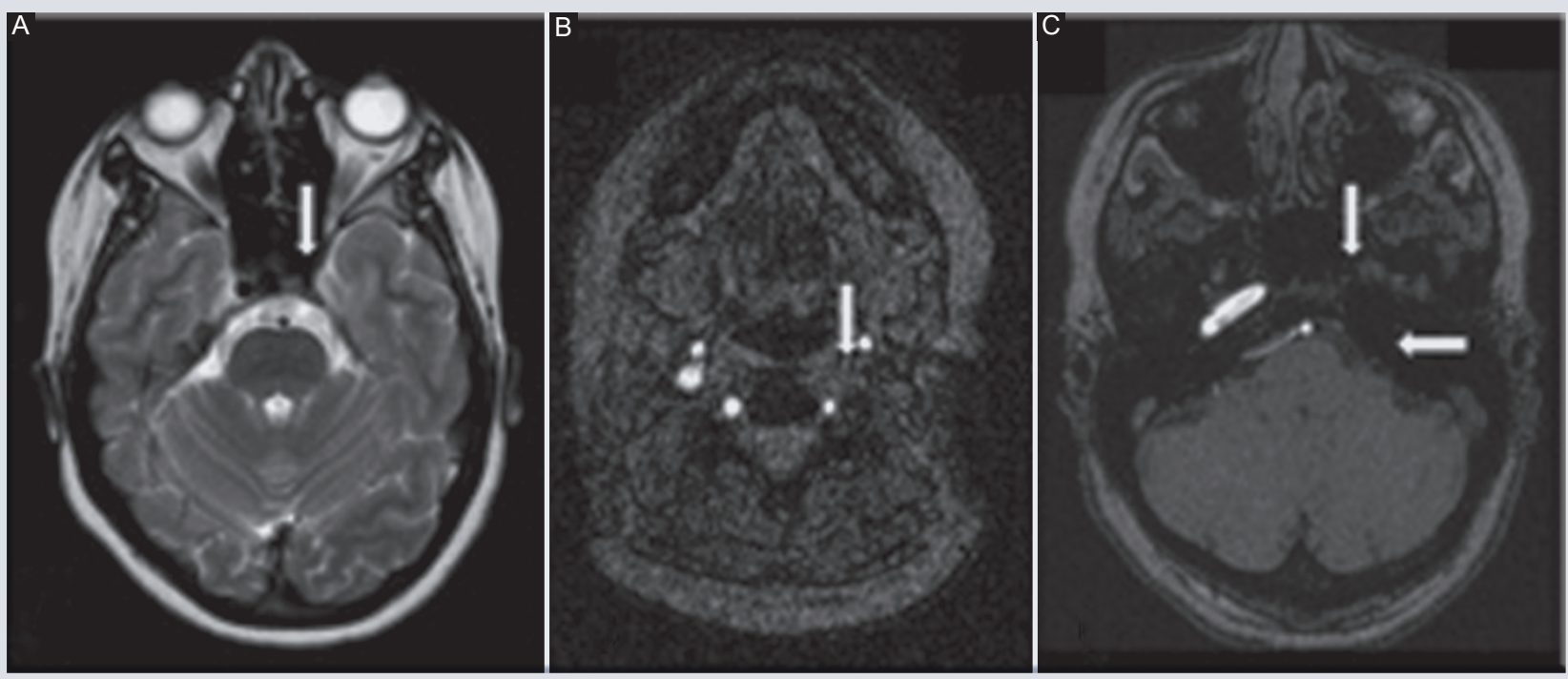

Figura 1. A: se identifica en secuencia T2 axial agenesia de arteria carótida interna (ACl) a nivel del seno esfenoidal (flecha). B: corte axial de angiorresonancia a nivel del cuello que muestra ausencia de contraste (flecha) de la ACl desde la bifurcación carotídea. C: corte axial de angiorresonancia a nivel de la roca del hueso temporal, donde se identifica la agenesia (flechas). (Cortesía del Dr. César Cardona).
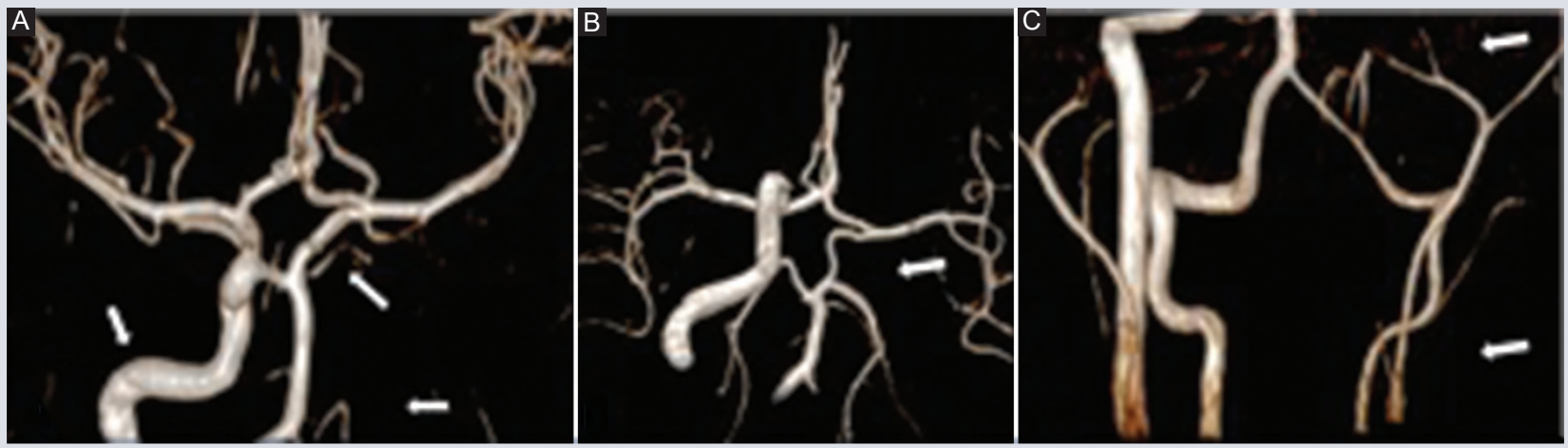

Figura 2: Reconstrucción en 3D de angiorresonancia. A: vista frontal en la cual se identifica una agenesia de arteria carótida interna ( $\mathrm{ACI}$ ) (flechas de la derecha) con llenado de la arteria cerebral anterio a través de la arteria comunicante anterior (ACoA) y de la arteria cerebral media a través de la arteria comunicante posterior ( $\mathrm{ACoP})$, además un notorio aumento de calibre de la $\mathrm{ACl}$ contralateral (flecha de la izquierda). B: vista caudal en la cual se identifican con mayor precisión la ACoA y la ACoP (la flecha señala la agenesia). C: agenesia de $\mathrm{ACl}$ desde la emergencia sobre la bifurcación carotídea. (Cortesía del Dr. César Cardona).

pueden llegar a presentarse síntomas como cefaleas recurrentes, visión borrosa, diplopía, epifora, hipoacusia, crisis epilépticas, síncopes, hemiplejia o hemiparesia, o hemorragia intracraneal; e inclusive enfermedades como aneurismas, glaucoma o síndrome de Horner. Durante una exploración física detallada, el principal signo que despertará la sospecha de una agenesia de ACI es la ausencia de pulso carotídeo.

Para una mayor comprensión es necesaria la retroalimentación de la anatomía vascular de 


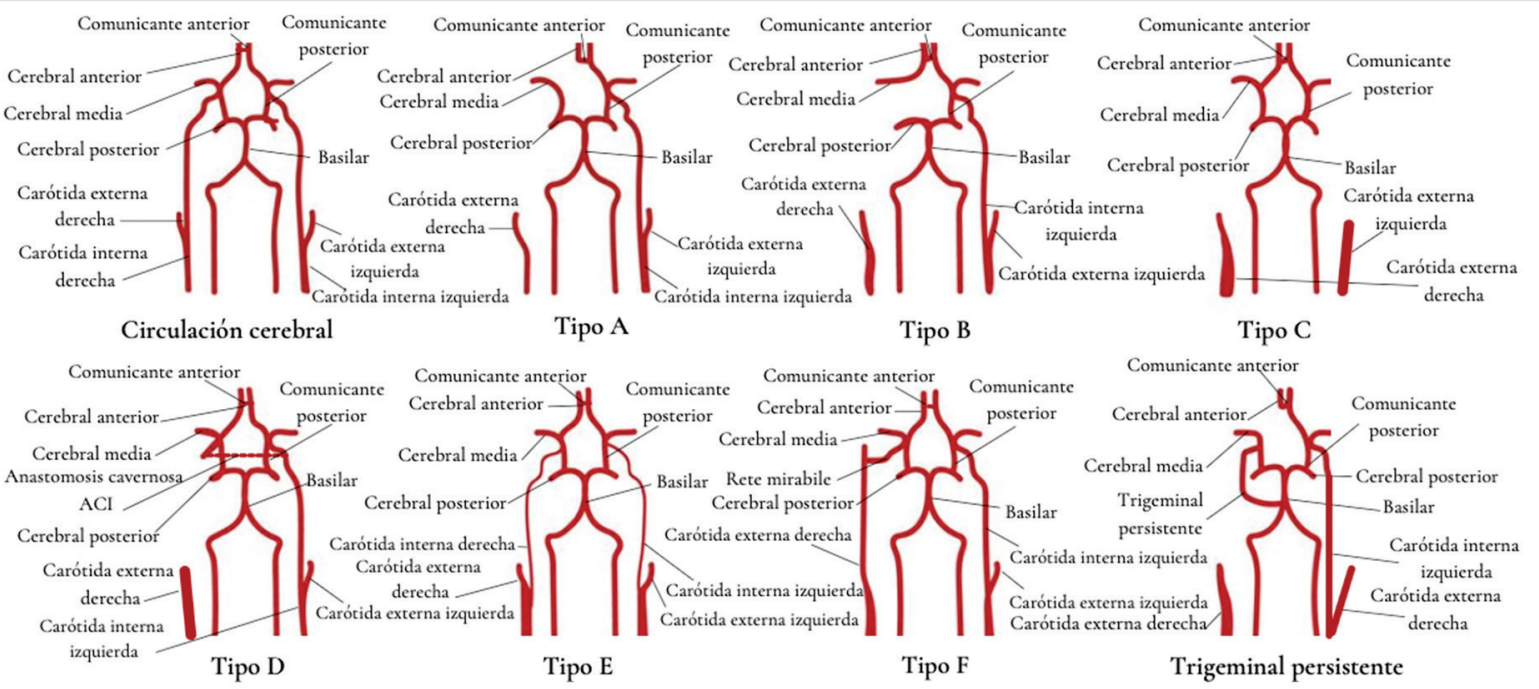

Figura 3. Patrones de Lie. Se observa la arteria persistente trigeminal. (Adaptado de: Flores-Silva et al, Li S et al, Escobar-Mallada et al).

esta arteria. A través del triángulo carotídeo (delimitado por el vientre superior del músculo omohioideo, el vientre posterior del músculo digástrico y el borde anterior del músculo esternocleidomastoideo) pasa la arteria carótida común, que suele palparse y auscultarse, la cual se divide a nivel del cartílago tiroides en la arteria carótida externa (ACE), y subsecuente al seno carotídeo (contiene el glomus carotídeo encargado de la quimiorrecepción), que es el barorreceptor, está la rama terminal que corresponde a la ACI. Se contiene junto con la vena yugular interna y posteriormente por el nervio vago dentro de la vaina carotídea ${ }^{6}$. Tras su entrada al espacio subaracnoideo por el canal petroso del hueso temporal, se divide en las ramas oftálmica, comunicante posterior (ACoP), coroidea, cerebral anterior (ACA) (que se ramifica en arterias corticales y centrales) y cerebral media $(\mathrm{ACM})$ (la rama más grande de la $\mathrm{ACI})^{7-9}$.

La ACI forma parte del polígono del Willis, por lo que su ausencia congénita producirá colateralidad o aumento del calibre de los vasos adyacentes. Lie y Hage establecieron la siguiente clasificación ${ }^{4}$ (Fig. 3):

- Patrón A: ausencia completa de la ACI de manera unilateral, por lo que la ACA homolateral se irriga desde la arteria comunicante anterior (ACoA) y de la ACM a través de la ACoP.

- Patrón B: ausencia completa de la ACI de manera unilateral, por lo que la ACA y la $\mathrm{ACM}$ se irrigan a través de la $\mathrm{ACI}$ contralateral a través de la ACoA.

- Patrón C: ausencia completa de la ACI de manera bilateral, por lo que la circulación dependerá de la ACoP.

- Patrón D: ausencia del segmento cervical (C1) de manera unilateral, por lo que la circulación dependerá de una adecuada comunicación intracavernosa desde la ACI contralateral.

- Patrón E: hipoplasia bilateral de la ACI; sin embargo, de ella nace la ACA. La ACM es sustituida por la ACoP. 


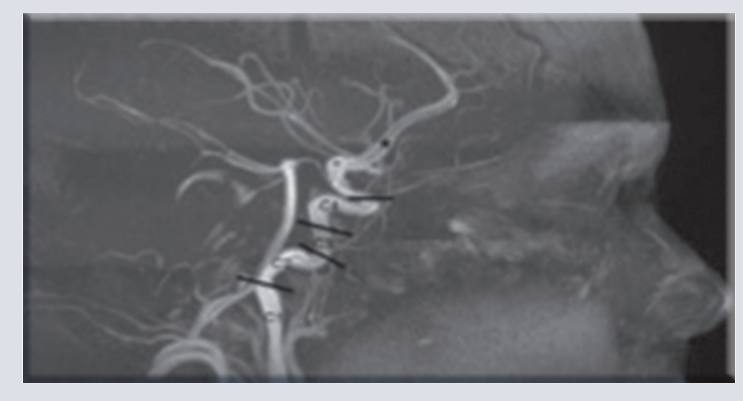

Figura 4. Angiorresonancia. Se observa segmentación de la arteria carótida interna derecha. (Imagen tomada de ref. 7).

- Patrón F: ausencia unilateral o bilateral de la ACI, con anastomosis transcraneales desde la ACE.

Existe la posibilidad de la persistencia y el posterior desarrollo de la arteria trigeminal persistente (Fig. 4), que se origina en la porción precavernosa (C4) de la ACI, que compensa la comunicación entre el sistema vertebrobasilar y el arterial carotídeo ${ }^{10}$, y que generalmente se encontrará hipertrófica en los patrones $\mathrm{A}, \mathrm{B}, \mathrm{C}$ y $\mathrm{E}$.

La clasificación más común es según la funcionalidad de la colateralidad desarrollada ${ }^{11,12}$ :

- Tipo fetal: el más frecuente, con ACA del lado homolateral afectada se irriga a través de la ACoA de la ACI contralateral, $y$ la ACM se irriga a través de la ACoP, rama de la ACM.

- Tipo adulto: la ACI contralateral irriga a través de la ACoA a la ACM y la ACA del hemisferio afectado.

- Tercer patrón: el hemisferio afectado es irrigado por anastomosis anómalas, tales como a) ACE a través de la arteria maxilar, oftálmica, vidiana o meníngea accesoria; b) porción C4 de la ACI contralateral a través de comunicaciones transelares intracavernosas; y c) vasos embrionarios persistentes.

Es importante tener en cuenta la segmentación carotídea debido a las ramas que nacen de cada una de estas, así como las hipoplasias que se puedan generar en alguna. Se compone de los siguientes siete segmentos: C1 o cervical, C2 o petroso, C3 o rasgado (o lacerum); C4 o cavernoso, C5 o clinoideo, C6 u oftálmico y C7 o comunicante (Fig. 4).

Sea cual sea el tipo o patrón, estos pacientes tienen una prevalencia del 25\% al 35\% de desarrollo de aneurismas cerebrales, aunque no se tienen reportes que indiquen la formación de estos previamente por otra etiología. La población general sin factores de riesgo ni angiopatía solo tiene un $2 \%$ a un $4 \%$ de probabilidades de desarrollarlos. La localización más frecuente es en la $\mathrm{ACoA}^{12}$.

La agenesia de ACI es un componente del síndrome PHACES (malformations of the Posterior fossa, Hemangioma, Arterial anomalies, Cardiopathy, Eye and Sternal anomalies).13,14. Además, por obvias razones, en enfermedades tromboembólicas es muy importante el diagnóstico, ya que los daños, cuando existe una agenesia de este tipo, son extensos.

Las pruebas de imagen adecuadas incluyen arteriografía, angiotomografía y angiorresonancia. La tomografía computarizada simple de cráneo puede ser útil para detectar la impermeabilidad del canal carotídeo de la porción petrosa. Llama la atención que durante usos habituales de ultrasonido carotídeo (incluyendo la modalidad Doppler) aparezca disminuido el pico sistólico de hasta $20 \mathrm{~cm} / \mathrm{s}$, 
con el consecuente aumento del flujo sanguíneo contralateral. De la misma manera, es viable descartar la enfermedad tromboembólica con esta técnica, ya que en un $25 \%$ puede encontrarse en manifestaciones clínicas por hemorragias intracraneales ${ }^{14}$. Los hallazgos radiológicos incluyen:

- Disminución del calibre o ausencia de visualización de la ACI de manera parcial o completa.

- Ausencia de permeabilidad del canal petroso del hueso temporal (altamente sugestivo).

- Presencia de colateralidad en el polígono de Willis, de arterias embrionarias persistentes o vasos transcraneales originados de la $\mathrm{ACE}^{14,15}$.

- Signo de la cuerda, muy sugestivo de hipoplasia de la ACI.

- Pico sistólico disminuido del lado afectado, de hasta $20 \mathrm{~cm} / \mathrm{s}$.

- Aumento del calibre o del grosor de capa íntima o muscular, o de ambas, de la arteria contralateral, así como de la arteria carótida común homolateral.

- Sistema compensatorio hipertrófico de arterias vertebrales.

El diagnóstico diferencial debe considerar fistulas carotídeo-cavernosas, compresión por tumoraciones y oclusión por ateromas o por enfermedad tromboembólica.

\section{CONCLUSIONES}

Claramente es un trastorno congénito con muy poco estudio debido a su infrecuencia, por lo que no existe una etiología clara; sin embargo, los pacientes que padecen una agenesia de este tipo suelen ser asintomáticos durante los primeros años de vida, e inclusive existe la teoría de que algunos que la padecieron jamás mostraron manifestaciones, aunque, claro está, aquellos con hábitos como mala alimentación y tabaquismo, que son más propensos a desarrollar enfermedad ateromatosa, también pueden ser más propensos a manifestar algún signo o síntoma.

\section{AGRADECIMIENTOS}

A los miembros del Departamento de Radiología del Hospital Ángeles Chihuahua.

\section{FINANCIAMIENTO}

Los autores declaran no haber recibido financiamiento para la realización de este artículo.

\section{CONFLICTO DE INTERESES}

Los autores declaran no tener conflictos de intereses.

\section{RESPONSABILIDADES ÉTICAS}

Protección de personas y animales. Los autores declaran que para esta investigación no se han realizado experimentos en seres humanos ni animales.

Confidencialidad de los datos. Los autores declaran que han seguido los protocolos de su centro de trabajo sobre la publicación de datos de pacientes.

Derecho a la privacidad y consentimiento informado. Los autores han obtenido el consentimiento informado de los pacientes y/o 


\section{sujetos referidos en el artículo. Este documen- to obra en poder del autor de corresponden-} cia.

\section{BIBLIOGRAFÍA}

1. Given CA $2^{\text {nd }}$, Huang-Hellinger F, Baker MD, Chepuri NB, Morris PP. Congenital absence of the internal carotid artery: case reports and review of the collateral circulation. Curtis A. Am J Neuroradiol. 2001;22:1953-9.

2. Scherle-Matamoros CE, Pérez-Nellar J, Roselló-Silva H, Rodríguez-Carballosa O, Hierro-García D. Hipoplasia-agenesia de la arteria carótida interna. Rev Ecuator Neurol. 2007;16:222-5.

3. González X, Landó F. Angiotomografía cerebral: variantes anatómicas más frecuentes del polígono de Willis. Ensayo iconográfico. Rev. Imagenol. 2014;27:93-7.

4. Flores-Silva F, Chiquete-Anaya E, Cantú-Brito C, Córdova-López P. Agenesia de la arteria carótida interna: presentación de dos casos y revisión de la literatura. Rev Ecuator Neurol. 2019;28:85-8.

5. Jiménez-Caballero PE. Agenesia de la arteria carótida interna y vertebral izquierdas. Neurol Arg. 2014;6:236-7.
6. Ruiz-Mercado H, Maldonado-Hernández H, Cruz-González JA. Hipoplasia de arteria carótida interna asociado con paraganglioma carotídeo. Reporte de un caso y revisión de la bibliografía. Cir Cir. 2014;82:302-8.

7. Acosta-Izquierdo L, Parra-Fuentes FA, Mora-Salazar JA, Tramontini-Jens C. Anatomía de la arteria carótida interna en angiotc y angiorresonancia. Revista Médica Sanitas. 2016;19:50-5.

8. Moore KL, Dalley AF, Agur AMR. Moore. Anatomía con orientación clínica. 8. ${ }^{\text {a }}$ ed. Barcelona: Wolters Kluwer; 2017. p. 1788-90.

9. Snell RS. Neuroanatomía clínica. 7. a ed. Barcelona: Wolters Kluwer; 2014. p. 794-5.

10. Pastore FA, Glenny P. Agenesia de carótida interna y riñón izquierdo. Cartas Científicas. 2018;83:224-5.

11. Li S, Hooda K, Gupta N, Kumar Y. Internal carotid artery agenesis: a case report and review of literature. Neuroradiol J. 2017;30:186-91.

12. Escobar-Mallada B, Benítez-Vázquez AM, Redondo-Buíl P, Meilán-Martínez A, Santamarta-Liébana E, Saiz-Ayala A. Ausencia congénita de la arteria carótida interna. European Society of Radiology, 2012. págs. 1-24. Disponible en: https:/ /epos.myesr.org/poster/esr/seram2012/S-0107

13. Campo CA, Manotas C, Galindo J, Acosta S. Síndrome de PHACE: reporte de un caso. Salud Uninorte. 2010;26:360-5.

14. da Silva-Neves W, Yochiharu-Kakudate M, Pereira-Cêntola C, GouveiaGarzon R, Poça-d'Água A, Sanches R. Agenesis of the internal carotid artery: a case report. Radiol Brasil. 2008;41:63-6.

15. Delgado-Sáiz S, Méndez LA, Gómez-Muga JJ, Sarmiento-De la Iglesia MM, Cisneros-Carpio S, Grande-Icarán D. Variantes anatómicas del polígono de Willis. Todo lo que debemos conocer. Radiología. 2016;58(Spec Cong):1438. 